\title{
A meta-analysis of Microarray Data is Effective for Identifying Gravity-Sensitive Genes
}

2 Yin Liang ${ }^{1}$, Mengxue Wang ${ }^{1}$, Yun Liu ${ }^{1}$, Chen Wang ${ }^{1}, K_{\text {Ken Takahashi }}{ }^{{ }^{*}}$, Keiji Naruse $^{1}$

$3{ }^{1}$ Department of Cardiovascular Physiology, Graduate School of Medicine, Dentistry and

4 Pharmaceutical Sciences, Okayama University, Okayama, Japan

$5 \quad *$ Correspondence:

6 Corresponding Author

7 takah-k2@okayama-u.ac.jp

8 Keywords: DNA microarrays, meta-analysis, microgravity, human umbilical vein endothelial 9 cells, prostaglandin transporter, clinostat

\section{Abstract}

11 Gravity affects the function and maintenance of organs, such as bones, muscles, and the heart. Several studies have used DNA microarrays to identify genes with altered expressions in response to gravity. However, it is technically challenging to combine the results from various microarray datasets because of their different data structures. We hypothesized it is possible to identify common changes in gene expression from the DNA microarray datasets obtained under various conditions and methods. In this study, we grouped homologous genes to perform a meta-analysis of multiple vascular endothelial cell and skeletal muscle datasets. According to the t-distributed stochastic neighbor embedding (t-SNE) analysis, the changes in the gene expression pattern in vascular endothelial cells formed specific clusters. We also identified candidate genes in endothelial cells that responded to gravity. Further, we exposed human umbilical vein endothelial cells to simulated microgravity using a clinostat and measured the expression levels of the candidate genes. Gene expression analysis using qRT-PCR revealed that the expression level of the prostaglandin transporter gene SLCO2A1 decreased in response to microgravity, consistent with the meta-analysis of microarray datasets. Furthermore, the direction of gravity affected the expression level of SLCO2A1, buttressing the finding that its expression was affected by gravity. These results suggest that a meta-analysis of DNA microarray datasets may help identify new target genes previously overlooked in individual microarray analyses.

\section{Introduction}

Long-term space missions, such as those to Mars, have become more feasible. During space missions, astronauts experience microgravity, which is the condition of being weightless. During spaceflight, the astronauts' cardiovascular function undergoes tremendous changes, such as blood redistribution, increased compliance with lower extremity veins, heart contraction, and orthostatic intolerance $[1,2]$. Thus, it is crucial to study the impact of spaceflight on various organs, such as the cardiovascular system. A recent study on twin astronauts demonstrated that a one-year spaceflight 
induced various changes, such as carotid artery distension and increased intima-media thickness [3]. This study suggests that retinal edema formation in space may be promoted by angiogenesis. Therefore, it is vital to elucidate the effect of microgravity on vascular endothelial cells for the coming space age.

Microgravity affects the morphology and physiology of the cardiovascular system. While the microgravity-induced changes are dramatic, they are thought to be mostly secondary to headward fluid shifts rather than direct effects of microgravity on the cardiovascular system [4]. Another factor that microgravity exerts on the orthostatic intolerance is a decrease in constrictor responses of mesenteric artery and vein [5].

On the other hand, the endothelium is one of the most sensitive tissues to changes in gravity $[6,7]$. Microgravity directly acts on vascular endothelial cells and affects processes, such as angiogenesis disorganization [10] and decreases the metabolism of the endothelial cells [11].

Several models, such as human subjects in bed rest, the suspended hind limbs of a rodent, clinorotated cultured cells, and humans, animals, and cultured cells in spaceflight [12-14], are used to evaluate the effect of microgravity on organs. On the other hand, DNA microarray is often performed using RNA extracted from various tissues to analyze the gene expressions affected by 53 microgravity.

However, the studies examining the effect of microgravity on gene expression tend to yield inconsistent results. Although many investigations generate gene expression data, only a few have cross-sectionally examined data, especially in space biology and medicine. Two factors make such comparisons of gene expression among multiple datasets difficult. First, the studies used different microarrays to analyze different gene sets. Second, the nomenclatures of the same genes differ in the studies.

We hypothesized that it was possible to identify common, essential microgravity-induced changes in gene expression from the results obtained using varying materials and methods. This study aimed to uncover the overlooked effects of microgravity on gene expression from accumulated valuable database resources by using different statistical methods. In this study, we applied a method developed by Kristiansson et al. [15] to categorize genes into homologous groups and perform a meta-analysis to resolve the problem of genes having different names in various DNA arrays. Since microgravity causes not only cardiovascular deconditioning but also muscle atrophy, we analyzed the changes in gene expression in endothelial cells and skeletal muscle obtained from microarray datasets in multiple simulated or in-flight microgravity experiments.

It would also be fascinating and important to identify the similarity in the impact of microgravity on used, it is difficult to perform a consistent analysis of the genes that respond to microgravity. To our knowledge, the similarity between the results from gene expression analysis obtained with different materials and methods has never been shown systematically in space biology. Here, we demonstrated such similarity by applying the t-distributed stochastic neighbor embedding (t-SNE) analysis [16], a method extensively used for visualizing high-dimensional data from flow cytometry 
Furthermore, after the gravity-sensitive genes were identified from the meta-analysis, their change in expression in human umbilical vein endothelial cells (HUVECS) was induced by simulated microgravity (SMG) using a clinostat and tested with qRT-PCR.

We found that the levels of $\angle O X, S L C O 2 A 1$, and TXNIP expression were significantly altered in response to SMG. Also, we found that the direction of gravity affected the expression of SLCO2A1. Instead of being specific to the condition of microgravity, the expression of SLCO2A1 may be altered by gravity from a direction different from the arrangement of its cytoskeleton. These results suggest altered expression in response to gravity changes. This strategy could potentially contribute to the advancement of space biology and medicine.

\section{$2 \quad$ Materials and methods}

\subsection{Microarray analysis}

The gene expression data for endothelial cells were obtained from two studies, Gene Express Omnibus (GSE43582) [7] and Ma et al. [17] (Table 1), which investigated the effects of microgravity. Ma et al. made two comparisons. It compared the $1 \mathrm{G}$ and SMG adherent groups, and between the $1 \mathrm{G}$ and SMG three-dimensional (3D) aggregate groups on day 7 . Then, we performed a metaanalysis to test the null hypothesis that a gene of interest was not differentially expressed among all genes in the databases using a method described by Kristiansson et al. [15]. The cross-experimental p-values were corrected for multiple testing using the false discovery rate (FDR) determined via the Benjamini-Hochberg procedure. For the muscle samples, gene expression data from 12 experiments investigating the effects of microgravity were obtained from Gene Express Omnibus (Table 2). We performed the same meta-analysis and p-value correction for endothelial cells.

\section{2 t-SNE analysis}

A t-SNE analysis was performed using R's tsne package (https://cran.rproject.org/web/packages/tsne/) to group the multiple DNA array datasets. The perplexity parameter in the t-SNE analysis indicates the effective number of neighbors, and the result of the tSNE plot may differ depending on its setting. The grouping by t-SNE analysis is shown to the same regardless of the value of perplexity by performing the t-SNE analysis with varying perplexity parameter 1, 2, or 3. In this study, the t-SNE analysis was performed at least 10 times at each perplexity parameter to confirm that the results showed a constant pattern. Furthermore, cluster analysis was performed using cluster 3.0 to group multiple DNA array datasets by a method different from the above t-SNE analysis [18].

\subsection{HUVEC culture} USA), $1 \times 10^{5}$ cells were cultured at $37^{\circ} \mathrm{C}$ at $5 \% \mathrm{CO}_{2}$ for the duration of the experiment.

\section{$114 \quad 2.4 \quad$ Exposure to SMG}


115

116

117

118

119

120

121

122

123

124

125

126

127

128

129

130

131

132

133

134

135

136

137

138

139

140

141

142

143

144

145

146

147

148

149

150

151

152

The HUVECs were seeded in the flasks and cultured for 2 days at $37^{\circ} \mathrm{C}$ at $5 \% \mathrm{CO}_{2}$ to ensure the cells attach to the bottom of the flasks. Then, the flasks were split into two groups, SMG and $1 \mathrm{G}$ control. The SMG flasks were clinorotated for 7 days using a clinostat (Zeromo; Kitagawa Iron Works Co. Ltd., Hiroshima, Japan) to achieve $10^{-3} \mathrm{G}$ in the incubator (Fig. 4A). During clinorotation, air bubbles were removed from the flasks to prevent shear stress. The $1 \mathrm{G}$ control flasks were placed next to the clinostat and kept static for 7 days.

\subsection{Cell culture under different gravitational configurations}

The HUVECs were seeded in the flasks simultaneously and cultured for 2 days to confirm the cells' attachment. Then, the flasks were divided into the "up," "side," and "down" configures, where the cells were on a horizontal surface, on a vertical surface, and hung on a horizontal surface, respectively (Fig. 7A). The "up" configuration was the control condition. This experiment was performed independently from the clinostat experiment in Section 2.4.

\subsection{Phase-contrast images of HUVECs}

For the SMG experiments, the cells' condition was observed by acquiring daily phase-contrast images of the same areas in the culture flask using a TE2000 inverted microscope. For the experiment involving different gravitational configurations, the same areas in the culture flask were imaged on the day before exposure to the gravitational conditions and after 7 days of exposure to the conditions to observe the morphology and growth of the cells.

\section{7 qRT-PCR}

Total RNA was harvested from cells using a High Pure RNA Isolation Kit (Roche Diagnostics Indianapolis, IN, USA), according to the manufacturer's protocols. The RNA concentration was measured using a spectrometer (Nanodrop ND-1000, Thermo Fisher Scientific, USA). RNA samples with an OD $260 / 280$ ratio of more than 1.80 were used for subsequent procedures. The samples were stored at $-80^{\circ} \mathrm{C}$.

For qRT-PCR, RNA samples were first heated at $70^{\circ} \mathrm{C}$ for 5 min to destroy the secondary structures. Then, the 1 ng of RNA was reverse-transcribed into cDNA using a Verso cDNA Synthesis Kit (Thermo Scientific, Lithuania, USA). The cDNA was used for qRT-PCR to quantify the expression of 20 genes (Table 3) using forward and reverse primers from previous publications or designed using the Primer3 (Version 0.4.0, http://bioinfo.ut.ee/primer3-0.4.0/) software. SYBR Green reagents (Life Technologies) were used for the relative quantification of RNA levels according to the manufacturer's instructions. Lastly, 18S rRNA was used as an internal control.

\subsection{Statistical analysis}

Statistical analysis was performed using Prism (version 6.07, GraphPad Software, La Jolla, CA, USA). The data were expressed as mean \pm SD. The significance of the differences between the experimental groups was determined using the Student's t-test or Kruskal-Wallis test with Dunn's Multiple Comparison. A p-value of 0.05 or less was considered statistically significant. 
153

154

155

156

157

158

159

160

161

162

163

164

165

166

167

168

169

170

171

172

173

174

175

176

177

178

179

180

181

182

183

184

185

186

187

188

189

190

191

192

193

194

\subsection{Statistical meta-analysis of gene expression in endothelial cells in response to microgravity conditions}

We analyzed the gene expression data from microgravity exposure experiments performed in spaceflight or with clinorotation in the laboratory (Table 1) to identify genes in endothelial cells with altered expression in response to microgravity. A total of 20,358 genes were retrieved from the spaceflight experiment, and 903 genes were retrieved from the clinorotation experiment. At first, GEO2R [19] was used to identify the genes differentially expressed in the ground control and flight samples. The $p$-values obtained from this analysis were used with the $p$-values from the comparison between the $1 \mathrm{G}$ and clinorotated adherent groups provided by Ma et al. $[X]$ for the subsequent meta-analysis. Applying the method described by Kristiansson et al. [X], 108 genes with a $p$-value of 0.001 or less were identified, of which 46 genes had a FDR of 0.05 or less (Fig. 1A). We randomly chose 10 genes from the 46 genes for subsequent gene expression assays using qPCR. Of the 10 genes, CCN5, encoding WISP2 (WNT1 Inducible Signaling Pathway Protein 2), had the lowest p-value of $2.13 \times 10^{-8}$, also the point with a high $-\log _{10}$ FDR value in the volcano plot (Figs. $1 B$ and $1 C$ ). $L O X$ (encoding lysyl oxidase) and MT1X (encoding metallothionein $1 X$ ) showed higher fold change values $(\operatorname{logFC})$ at 4.11 and 1.71, respectively, in clinorotated EA.hy926 cells, indicating that microgravity facilitated the changes in their expression (Fig. 1C). However, the logFC values of $L O X$ and MT1X were -0.35 and -0.45 , respectively, in the space-flown HUVECs, indicating the decreased expression of these genes. The logFC value of $S L C O 2 A 1$ (encoding solute carrier organic anion transporter family member 2A1) was -2.10 in the clinorotated EA.hy 926 cells and -0.23 in the space-flown HUVECS, with an FDR of $5.18 \times 10^{-2}$.

Then, we performed another meta-analysis using the same $p$-values from the space-flown HUVECS and those from another microgravity condition, the 3D aggregates in clinorotated EA.hy 926 cells. As a result, 79 genes with a $p$-value of 0.001 or less were identified, of which 64 genes had a FDR of 0.05 or less (Fig. 2A). Again, LOX showed a significant $p$-value of $2.95 \times 10^{-7}$ and a high logFC value of 3.44 in the clinorotated EA.hy926 cells, with the logFC of -0.35 , in opposite direction in the spaceflown HUVECs (Figs. 2A-C). In contrast, SLCO2A1 showed a significant logFC value of -2.66 and an FDR of $2.60 \times 10^{-3}$, with a logFC of -0.23 , in the same direction in the space-flown HUVECs.

\subsection{Statistical meta-analysis of gene expression in skeletal muscle in response to microgravity conditions}

Next, we analyzed the gene expression data from the experiments using multiple microgravity loads on skeletal muscle (Table 2). The $p$-values were obtained using GEO2R from each dataset. For example, the differential expression of genes across pre and post-bed rest experiments in the soleus muscle was analyzed using the GSE14798 dataset. A meta-analysis of 12 datasets identified 6,328 genes with a FDR of 0.05 or less, of which 10 genes exhibited changes in expression in the same direction (both increasing or decreasing) in most of the datasets with valid fold change (FC) values (Fig. 3). These genes were selected for the subsequent gene expression assays using qPCR.

Then, we performed the t-SNE analysis to gain insight into the similarity between the DNA array datasets. We analyzed 3,290 genes with valid FC values in all the datasets listed in Table 2 . The individual datasets were marked A-L. The cost function in the t-SNE analysis reflected the difference between the distance of each gene in multidimensions, 3,290 dimensions in this case, and that in 2D. After performing the t-SNE analysis, the cost value converged between 1,000 and 
195

196

197

198

199

200

201

202

203

204

205

206

207

208

209

210

211

212

213

214

215

216

217

218

219

220

221

222

223

224

225

226

5,000 steps (Fig. 4A). In step 10,000, the cost value converged to $0.480,0.293$, and 0.259 when the perplexity parameter was 1,2 , and 3 , respectively. When the perplexity was 1 , the array datasets were divided into two groups, i.e., $A / B / C / D / E / I / J$ and F/G/H/K/L (Fig. 4B). A similar tendency was observed when the perplexity was 2 and 3 (Figs. 4C and 4D). Furthermore, a similar pattern was observed in the results of the cluster analysis (Fig. 4I).

It would also be interesting to see how the microgravity-induced changes in the gene expression pattern in endothelial cells differ from those in muscle tissue. We investigated this by attempting to perform the t-SNE analysis by combining the 2 array datasets obtained from the endothelial cells ("X" and "Y" in Table 1) with the 12 array datasets obtained from skeletal muscle ("A-L" in Table 2). However, only 52 genes with valid FC values were identified in the combined datasets with 14 arrays. The removal of datasets $A$ and $B$ from the combined datasets resulted in the identification of 177 genes with valid FC values. We conducted the t-SNE analysis using these 177 genes. As a result, the cost value converged within 700 steps (Fig. 4E). In step 10,000, the cost value converged to $0.488,0.321$, and 0.206 when the perplexity was 1,2 , and 3 , respectively. When the perplexity was 1 , the array datasets were divided into 3 groups: $C / F / H / I / J / X / Y, D / E$, and $G / K / L$ (Fig. $4 F$ ). A similar pattern was observed when the perplexity was 2 and 3 (Figs. $4 \mathrm{G}$ and $4 \mathrm{H}$ ); the endothelium-derived datasets $X$ and $Y$ were relatively close to each other and within the same group.

\subsection{Morphological analysis of HUVECs under SMG using a clinostat}

The shape of the cells in the SMG was compared to those in the $1 \mathrm{G}$ control groups after exposure to gravitational conditions. The groups exhibited similar cell shapes. Also, the cell numbers in the groups were similar, with $622.33 \pm 264.66$ in the SMG group and $563 \pm 101.60$ in the $1 \mathrm{G}$ control group ( $p>0.05$ ) (Fig. 5B-D).

\subsection{Comparison of mRNA expression between control and SMG conditions by qRT-PCR}

After exposing the HUVECs to different gravity conditions, we measured the mRNA level of the 10 genes selected from the DNA array meta-analysis of endothelial cells (Fig. 6A). LOX expression was higher in the SMG group than in the $1 \mathrm{G}$ group at $1.91 \pm 0.27$ and $1.20 \pm 0.16$, respectively $(p<0.05)$. Conversely, SLCO2A1 (SMG, 0.43 $\pm 0.12 ; 1 G, 0.94 \pm 0.05 ; p<0.01$ ) and TXNIP expression (SMG, 0.43 $\pm 0.10 ; 1 G, 0.96 \pm 0.06 ; p<0.01)$ was lower under the SMG condition than the $1 \mathrm{G}$ condition.

Next, we measured the expression of the 10 genes selected from the DNA array meta-analysis of muscle tissue (Fig. 6B). In contrast to the result from genes selected from the meta-analysis of endothelial cell DNA arrays, there were no statistically significant differences in gene expression between the SMG and $1 \mathrm{G}$ groups.

\subsection{Morphological analysis of HUVECs under different gravitational configurations}

We tested the effects of the direction of gravity on the morphology and growth of endothelial cells by incubating HUVECs in flasks at the up, side, or down configuration (Fig. 7A). In the down configuration, the cells were less dense than the cells in the up and side configurations (Figs. 7B-D). Moreover, the cells in the down configuration appeared "brushed" and "branched" (Fig. 7D). The number of cells was the smallest in the down configuration at $209.4 \pm 112.6$ and largest in the side configuration at $638.6 \pm 349.4$ (Fig. 7E). The difference in cell numbers between the down and side configurations was statistically significant $(p<0.05)$. 
bioRxiv preprint doi: https://doi.org/10.1101/2021.03.31.437982; this version posted April 2, 2021. The copyright holder for this preprint (which was not certified by peer review) is the author/funder, who has granted bioRxiv a license to display the preprint in perpetuity. It is made available under aCC-BY 4.0 International license.

\subsection{Comparison of mRNA expression among the up, side, and down configurations by qRT- PCR}

After exposing HUVECs to different gravity configurations, we measured the expression of the 10 genes selected from the DNA array meta-analysis of endothelial cells (Fig. 8). Kruskal-Wallis test revealed that gravity configuration affected the expression of $S L C O 2 A 1$, with a statistically significant difference (Dunn's post-hoc test, $p<0.05$ ) in expression between the side and down configurations.

\section{Discussion}

Researchers have used different types of species, organs, and conditions of gravity to evaluate microgravity's effect. However, it is challenging to extract meaningful knowledge from these results. In this study, we performed a meta-analysis of gene expression using DNA microarray datasets obtained from experiments performed on diverse samples and species using various methods to mimic or achieve microgravity.

Interestingly, the t-SNE analysis revealed that gene expression patterns were grouped in DNA array datasets. For example, the datasets $(\mathrm{G}, \mathrm{H}, \mathrm{K}$, and $\mathrm{L})$ obtained from the skeletal muscle of space-flown mice formed a different cluster from datasets (A, B, C, D, E, and J) from skeletal muscle under simulated microgravity, except that one dataset from the space-flown sample (I) was in the "simulated microgravity" cluster and another dataset from simulated microgravity (F) was in the "spaceflight" cluster (Figs. 4B-D). Meanwhile, the datasets from hind limb unloading (D and E) were located far from the "spaceflight" cluster. This tendency was also observed when the number of analyzed genes was reduced to 177 (Figs. 4F-H). However, it should also be noted that the difference in experimental conditions between the datasets $\mathrm{G} / \mathrm{H} / \mathrm{K} / \mathrm{L}$ and the datasets $\mathrm{D} / \mathrm{E}$ was recognized not only in the mode of microgravity but also in the duration of microgravity and the method of sample storage. Nevertheless, according to the t-SNE analysis, the gene expression pattern in datasets D and E differed from G, K, and L. This fact was also supported by the result of cluster analysis (Fig. 4I).

In addition, the DNA array datasets obtained from endothelial cells under actual $(X)$ and simulated (Y) microgravity are located close to each other (Figs. 4F-H), suggesting a remarkable similarity in gene expression patterns, given that the datasets come from independent research groups. This result provides a valid basis for estimating gene expression change under microgravity in space with simulated microgravity datasets in endothelial cells.

Next, we investigated the genes whose expression may be affected by microgravity. The study results demonstrated that SLCO2Al expression was decreased in both space-flown HUVECs (FC value = -0.23 ) and clinorotated EA.hy926 cells (FC value = -2.66) (Fig. 2A). Furthermore, the qRT-PCR analysis revealed that $S L C O 2 A 1$ expression was decreased in response to a 7-day exposure to SMG using a clinostat, in parallel to the results in space-flown HUVECs and clinorotated EA.hy926 cells (Fig. 6). Importantly, the decreased expression of SLCO2A1 was not found in previous studies using space-flown HUVECs [7] and clinorotated EA.hy926 cells [17]. In other words, the decreased expression of $S L C O 2 A$ has been identified via a meta-analysis of multiple sets of previously published data.

Other genes responsive to microgravity have also been uncovered, such as LOX, encoding LOX with a copper-dependent amine oxidase activity and catalytic activity in the cross-linking of collagen and 
277

278

279

280

281

282

283

284

285

286

287

288

289

290

291

292

293

294

295

296

297

298

299

300

301

302

303

304

305

306

307

308

309

310

311

312

313

314

315

316

317

318

elastin [20], was affected. $L O X$ expression was increased, consistent with the results in EA.hy926 cells [17]. However, the expression of LOX is reduced in space-flown HUVECs [7]. Such discrepancy may be due to the differences in cell types and the conditions of microgravity, spaceflight vs. clinorotation. Another gene whose expression is altered by gravity changes is LRP4, which is involved in forming and maintaining neuromuscular junctions [21]. Interestingly, the expression of LRP4 was increased in this meta-analysis (Fig. 3). It is tempting to speculate that one of the mechanisms of muscle atrophy under microgravity is the altered expression of LRP4; this hypothesis should be carefully studied in the future.

On the other hand, we analyzed the genes with identified sensitivity to gravity in the context of skeletal muscle but observed no changes in their expression in HUVECs exposed to SMG. When we analyzed the genes that showed sensitivity to gravity in skeletal muscle, no changes were observed in the gene expression level in HUVEC underwent SMG (Fig. 6B). This result suggests that modulation of gene expression is organ-specific. This is also suggested because the two DNA array datasets from endothelial cells are located outside of the muscle cluster in the t-SNE analysis.

Furthermore, the direction of gravity was found to affect the morphology, proliferation, and gene expression of HUVECs in this study. The decrease in the cell number in the "down" configuration is likely due to the change in the cell adhesion in response to gravity. However, the cells are unlikely to simply fall off, as the adhesion of extracellular matrix proteins to the culture flask is relatively stable. Cells are known to respond to gravity. In animals, the direction of gravity affects the proliferation of osteoblasts [22]. Interestingly, in our study, osteoblast proliferation is inhibited in the inverted culture, similar to the HUVECs cultured in the down configuration. On the other hand, plant cells respond to gravity's direction, thus modulating the direction of root growth [23]. In plants, it is thought that the sedimentation of heavy statolith increases the tension of the actin cytoskeleton and subsequently activates mechanosensitive ion channels [24]. Suppose heavy nuclei and mitochondria also contribute to an animal cell's gravity sensing mechanism similar to plant statolith. In that case, this mechanism may explain the change in gene expression depending on the direction of gravity observed in the current study.

Intriguingly, SLCO2Al's expression was modulated in response to the direction of gravity in this study. In line with the results from previous studies using SMG, gravity appears to affect the expression of $S L C O 2 A 1$, which encodes a prostaglandin transporter with a recently discovered involvement in the regulation of body temperature [25]. Although it is tempting to speculate that the altered thermoregulation in astronauts [26] is partly attributed to the altered expression of the $S L C O 2 A 1$, this hypothesis should be tested in the future.

\section{Conclusion}

Using the meta-analysis of DNA microarray datasets obtained from the vascular endothelial cells, we have identified gene candidates whose expression levels change under microgravity. Accordingly to the t-SNE analysis, among the mixed datasets from endothelial cells and skeletal muscle, the gene expression patterns in response to gravity in endothelial cells form specific clusters.

Experiments using endothelial cells under simulated microgravity and different directions of gravity confirmed that the expression of one of the candidate genes, SLCO2A1 encoding the prostaglandin transporter, is indeed altered. These results suggest that meta-analysis of multiple microarray data is useful for identifying novel target genes. 
321 The authors declare that the research was conducted in the absence of any commercial or financial relationships that could be construed as a potential conflict of interest.

323

\section{Author Contributions}

KT and KN contributed to the conception and design of the study; YIL and MW performed the experiments; MW, YIL, YUL, CW, and KT wrote sections of the manuscript. All authors contributed to manuscript revision, and all authors read and approved the submitted version.

\section{$7 \quad$ Funding}

330 This study was supported by a Grant-in-Aid for Scientific Research on Innovative Areas [No.

331 15H05936].

332

3338 Abbreviations

FC Fold change

335 HUVEC

Human umbilical vein endothelial cells

336 SMG Simulated microgravity

337 t-SNE

t-distributed stochastic neighbor embedding

\section{Acknowledgments}

340 The authors gratefully acknowledge Central Research Laboratory, Okayama University Medical 341 School for the assistance of qRT-PCR, Dr. Hitoshi Osada for preliminary research of this work, and Dr. Masatoshi Morimatsu for his valuable suggestions.

Figure 1. A meta-analysis of gene expression in endothelial cells in response to microgravity conditions.

(A) Top 46 genes with the smallest $p$-values (FDR < 0.05), which were identified from the DNA array analyses of human umbilical vein endothelial cells (HUVEC) and EA.326hy cells under spaceflight and simulated microgravity conditions, respectively. Additional genes with relatively low $p$-values were listed below the top 46 genes. HU_FC: Log fold change value in HUVEC. EA_FC: Log fold change value in EA.hy 926 cell. 
Figure 2. A meta-analysis of gene expression in endothelial cells in response to other microgravity conditions.

(A) Top 64 genes with the smallest $p$-values (FDR $<0.05$ ), which were identified from the DNA array analyses of human umbilical vein endothelial cells (HUVEC) and EA.326hy cells under spaceflight and simulated microgravity conditions, respectively. Additional genes with relatively low $p$-values were listed below the top 46 genes. HU_FC: Log fold change value in HUVEC, EA_FC: Log fold change value in EA.hy926 cell. cells. Horizontal dotted line: a line at which FDR is equal to 0.05. Vertical dotted line: a line at which $\log F C$ is equal to zero.

Figure 3. A meta-analysis of gene expression in muscle samples in response to microgravity conditions. The 10 genes exhibiting changes in expression in the same direction (all increasing or decreasing) in most of the datasets were listed. vastus I: vastus lateralis. gcnemius: gastrocnemius. EDL: extensor digitorum longus. longis: longissimus dorsi.

Figure 4. The contribution of experimental conditions to the gene expression pattern. (A-D) The t-SNE analysis of the DNA array datasets obtained from skeletal muscle (Table 2). (A) The multidimensions and that in $2 \mathrm{D}$, over time at the perplexity of 1,2 , and 3 . The t-SNE plots at perplexity $=1(B), 2(C)$, and $3(D)$ are shown. (E-H) The t-SNE analysis of DNA array datasets obtained from endothelial cells (Table 1) and skeletal muscle (Table 2). (E) The convergence of cost values over time at the perplexity of 1,2 , and 3 . The $t-$ SNE plots at perplexity $=1(F), 2(G)$, and $3(H)$ are shown. Dashed lines are drawn to guide the grouping of datasets. (I) The cluster analysis of DNA array datasets obtained from skeletal muscle (Table 2).

Figure 5. The morphological analysis of human umbilical vein endothelial cells (HUVECS) under simulated microgravity.

(A) The clinostat is installed in a $\mathrm{CO}_{2}$ incubator. The $1 \mathrm{G}$ control flasks were placed next to the clinostat (bottom right). Phase-contrast images of HUVECs after 3 days of exposure to $1 \mathrm{G}$ (B) and simulated microgravity (C). Marker: $200 \mu \mathrm{m}$. (D) The number of cells in the simulated microgravity conditions compared to those in the control condition after 7 days of culturing. Data were expressed as the mean \pm S.E.M.

Figure 6. The comparison of gene expression between the control and simulated microgravity conditions in human umbilical vein endothelial cells. (A) The gene sets were obtained from the meta-analyses of datasets from endothelial cells. (B) The gene sets were obtained from the meta-analyses of datasets from muscle samples. The relative quantity of each gene was measured using qRT-PCR and normalized to the level of $18 \mathrm{~S}$ rRNA. The data were expressed as the mean \pm S.E.M. *: $p<0.05, * *: p<0.01$.

401

402 
404 (A) The gravitational configurations of cell culture. Left, up configuration; middle, side configuration; 405 and right, down configuration. The phase-contrast images of HUVECs under the up (B), side (C), or 406 down (D) configuration on day 7. Marker: $200 \mu \mathrm{m}$. (E) The number of cells in the up, side, and down 407 configurations. The data were expressed as the mean \pm S.E.M. *: $p<0.05$.

408

409 Figure 8. The comparison of gene expression of the cells in the up, side, and down configurations.

410 The relative quantity of each gene was assayed using qRT-PCR and normalized to the $18 \mathrm{~S}$

411 rRNA level. The data were expressed as the mean \pm S.E.M. *: $p<0.05$. 
1. Delp, M. D.; Charvat, J. M.; Limoli, C. L.; Globus, R. K.; Ghosh, P., Apollo Lunar Astronauts Show Higher Cardiovascular Disease Mortality: Possible Deep Space Radiation Effects on the Vascular Endothelium. Sci Rep 2016, 6, 29901.

2. Tanaka, K.; Nishimura, N.; Kawai, Y., Adaptation to microgravity, deconditioning, and countermeasures. J Physiol Sci 2017, 67, (2), 271-281.

3. Garrett-Bakelman, F. E.; Darshi, M.; Green, S. J.; Gur, R. C.; Lin, L.; Macias, B. R.; McKenna, M. J.; Meydan, C.; Mishra, T.; Nasrini, J.; Piening, B. D.; Rizzardi, L. F.; Sharma, K.; Siamwala, J. H.; Taylor, L.; Vitaterna, M. H.; Afkarian, M.; Afshinnekoo, E.; Ahadi, S.; Ambati, A.; Arya, M.; Bezdan, D.; Callahan, C. M.; Chen, S.; Choi, A. M. K.; Chlipala, G. E.; Contrepois, K.; Covington, M.; Crucian, B. E.; De Vivo, I.; Dinges, D. F.; Ebert, D. J.; Feinberg, J. I.; Gandara, J. A.; George, K. A.; Goutsias, J.; Grills, G. S.; Hargens, A. R.; Heer, M.; Hillary, R. P.; Hoofnagle, A. N.; Hook, V. Y. H.; Jenkinson, G.; Jiang, P.; Keshavarzian, A.; Laurie, S. S.; Lee-McMullen, B.; Lumpkins, S. B.; MacKay, M.; Maienschein-Cline, M. G.; Melnick, A. M.; Moore, T. M.; Nakahira, K.; Patel, H. H.; Pietrzyk, R.; Rao, V.; Saito, R.; Salins, D. N.; Schilling, J. M.; Sears, D. D.; Sheridan, C. K.; Stenger, M. B.; Tryggvadottir, R.; Urban, A. E.; Vaisar, T.; Van Espen, B.; Zhang, J.; Ziegler, M. G.; Zwart, S. R.; Charles, J. B.; Kundrot, C. E.; Scott, G. B. I.; Bailey, S. M.; Basner, M.; Feinberg, A. P.; Lee, S. M. C.; Mason, C. E.; Mignot, E.; Rana, B. K.; Smith, S. M.; Snyder, M. P.; Turek, F. W., The NASA Twins Study: A multidimensional analysis of a year-long human spaceflight. Science 2019, 364, (6436).

4. Norsk, P., Adaptation of the cardiovascular system to weightlessness: Surprises, paradoxes and implications for deep space missions. Acta Physiol (Oxf) 2020, 228, (3), e13434.

5. Behnke, B. J.; Stabley, J. N.; McCullough, D. J.; Davis, R. T., 3rd; Dominguez, J. M., 2nd; Muller-Delp, J. M.; Delp, M. D., Effects of spaceflight and ground recovery on mesenteric artery and vein constrictor properties in mice. FASEB J 2013, 27, (1), 399-409.

6. Maier, J. A.; Cialdai, F.; Monici, M.; Morbidelli, L., The impact of microgravity and hypergravity on endothelial cells. Biomed Res Int 2015, 2015, 434803.

7. Versari, S.; Longinotti, G.; Barenghi, L.; Maier, J. A.; Bradamante, S., The challenging environment on board the International Space Station affects endothelial cell function by triggering oxidative stress through thioredoxin interacting protein overexpression: the ESASPHINX experiment. FASEB J 2013, 27, (11), 4466-75.

8. Cotrupi, S.; Ranzani, D.; Maier, J. A., Impact of modeled microgravity on microvascular endothelial cells. Biochim Biophys Acta 2005, 1746, (2), 163-8.

9. Carlsson, S. I.; Bertilaccio, M. T.; Ballabio, E.; Maier, J. A., Endothelial stress by gravitational unloading: effects on cell growth and cytoskeletal organization. Biochim Biophys Acta 2003, 1642, (3), 173-9.

10. Villa, A.; Versari, S.; Maier, J. A.; Bradamante, S., Cell behavior in simulated microgravity: a comparison of results obtained with RWV and RPM. Gravit Space Biol Bull 2005, 18, (2), 89-90.

11. Kapitonova, M. Y.; Muid, S.; Froemming, G. R.; Yusoff, W. N.; Othman, S.; Ali, A. M.; Nawawi, H. M., Real space flight travel is associated with ultrastructural changes, cytoskeletal disruption and premature senescence of HUVEC. Malays J Pathol 2012, 34, (2), 103-13.

12. Perhonen, M. A.; Franco, F.; Lane, L. D.; Buckey, J. C.; Blomqvist, C. G.; Zerwekh, J. E.; Peshock, R. M.; Weatherall, P. T.; Levine, B. D., Cardiac atrophy after bed rest and spaceflight. J Appl Physiol (1985) 2001, 91, (2), 645-53. 
460

461

462

463

464

465

466

467

468

469

470

471

472

473

474

475

476

477

478

479

480

481

482

483

484

485

486

487

488

489

490

491

492

493

494

495

496

497

13. Globus, R. K.; Morey-Holton, E., Hindlimb unloading: rodent analog for microgravity. $J$ Appl Physiol (1985) 2016, 120, (10), 1196-206.

14. Fukuda, S.; Iida, H., Effects of clinostat-microgravity on bone and calcium metabolism in rats. J Gravit Physiol 2000, 7, (2), P123-4.

15. Kristiansson, E.; Osterlund, T.; Gunnarsson, L.; Arne, G.; Larsson, D. G.; Nerman, O., A novel method for cross-species gene expression analysis. BMC Bioinformatics 2013, 14, 70.

16. van der Maaten, L. J. P.; Hinton, G. E., Visualizing High-Dimensional Data Using t-SNE. Journal of Machine Learning Research 2008, 9, 2579-2605.

17. Ma, X.; Wehland, M.; Schulz, H.; Saar, K.; Hubner, N.; Infanger, M.; Bauer, J.; Grimm, D., Genomic approach to identify factors that drive the formation of three-dimensional structures by EA.hy926 endothelial cells. PLoS One 2013, 8, (5), e64402.

18. de Hoon, M.; Imoto, S.; Nolan, J.; Miyano, S., Open Source Clustering Software.

Bioinformatics 2004, 20, (9), 1453-1454.

19. Smyth, G. K., Linear models and empirical bayes methods for assessing differential expression in microarray experiments. Stat Appl Genet Mol Biol 2004, 3, Article3.

20. Vallet, S. D.; Ricard-Blum, S., Lysyl oxidases: from enzyme activity to extracellular matrix cross-links. Essays Biochem 2019, 63, (3), 349-364.

21. Barik, A.; Lu, Y.; Sathyamurthy, A.; Bowman, A.; Shen, C.; Li, L.; Xiong, W. C.; Mei, L., LRP4 is critical for neuromuscular junction maintenance. J Neurosci 2014, 34, (42), 13892905.

22. Kacena, M. A.; Todd, P.; Gerstenfeld, L. C.; Landis, W. J., Experiments with osteoblasts cultured under varying orientations with respect to the gravity vector. Cytotechnology 2002, 39, (3), 147-54.

23. Herranz, R.; Medina, F. J., Cell proliferation and plant development under novel altered gravity environments. Plant Biol (Stuttg) 2014, 16 Suppl 1, 23-30.

24. Tatsumi, H.; Furuichi, T.; Nakano, M.; Toyota, M.; Hayakawa, K.; Sokabe, M.; Iida, H., Mechanosensitive channels are activated by stress in the actin stress fibres, and could be involved in gravity sensing in plants. Plant Biol (Stuttg) 2014, 16 Suppl 1, 18-22.

25. Nakamura, Y.; Nakanishi, T.; Shimada, H.; Shimizu, J.; Aotani, R.; Maruyama, S.; Higuchi, K.; Okura, T.; Deguchi, Y.; Tamai, I., Prostaglandin Transporter OATP2A1/SLCO2A1 Is Essential for Body Temperature Regulation during Fever. J Neurosci 2018, 38, (24), 55845595.

26. Stahn, A. C.; Werner, A.; Opatz, O.; Maggioni, M. A.; Steinach, M.; von Ahlefeld, V. W.; Moore, A.; Crucian, B. E.; Smith, S. M.; Zwart, S. R.; Schlabs, T.; Mendt, S.; Trippel, T.; Koralewski, E.; Koch, J.; Chouker, A.; Reitz, G.; Shang, P.; Rocker, L.; Kirsch, K. A.; Gunga, H. C., Increased core body temperature in astronauts during long-duration space missions. Sci Rep 2017, 7, (1), 16180. 
2240

19513

9710

2833

11050

15076

9725

2611

16295

19108

3620

15144

9718

13174

14083

7980

10769

6392

6249

1551

16615

10221

282

14474

14464

15059

5792

259

17800

9795

1370

5366

19386

2923

3652

6248

2794

7093

17733

5250

8606

17659

18893

6924

15790

16292

3586

18369

17820

4773
$P$ value

FDR

HUFC

EA FC

CA9

3.91E-10 8.00E-06

$1.06 \mathrm{E}-08 \quad 1.09 \mathrm{E}-04$

2.13E-08 1.46E-04

LOX

4.24E-08

$\mathrm{CDH} 15$

2.17E-04

MT1X

23E-07

RPL9

$1.32 \mathrm{E}-07$
$3.94 \mathrm{E}-07$

LPCAT2

CCL14

6.59E-07

SLCO4A1

7.63E-07

UPK1A

9.63E-07

CPA4

1.19E-06

RPS7

1.37E-06

LPAR1

1.50E-06

PIK3C2B

1.73E-06

$\mathrm{PSPH}$

$2.28 \mathrm{E}-06$

INSM2

2.78E-06

MMP2

2.84E-06

3.63E-06

GADD45B

BGN

4.02E-06

$5.89 \mathrm{E}-06$

SNORD14E 6.34E-06

MCAM

ADAMTS1

7.12E-06

RASSF2

8.02E-06

RASGRP3

1.23E-05

RPL37

1.51E-05

FCN3

1.74E-05

ADAM15

1.75E-05

TGFBI

LRRC33 1.76E-05 1.89E-05

2.12E-05 AXL 2.36E-05

FAM107A 2.66E-05 VWF 3.23E-05

CEACAM1 5.39E-05 CPS1

$5.41 \mathrm{E}-05$

GADD45A

5.76E-05

CDC25B

HBG1

TESC

ETS2

KLRK1

TCN2

TXNIP

GSN

6.07E-05

$6.61 \mathrm{E}-05$

$6.85 \mathrm{E}-05$

7.02E-05

8.27E-05

9.64E-05

9.72E-05

SHANK3

9.81E-05

SLCO2A1

CORO2A

TNS3

THAP1

DYSF

$129 \mathrm{E}-04 \quad 5.18 \mathrm{E}-02$

1.98E-04 6.64E-02

2.86E-04 8.58E-02

2.89E-04 8.58E-02

3.95E-04 1.06E-01
$-0.42 \quad 2.70$

$\begin{array}{ll}-0.41 & 2.21\end{array}$

$-0.13$

$-0.35$

$-0.21$

$-0.45$

$1.15 \mathrm{E}-03$

1.69E-03

$1.74 \mathrm{E}-03$

.79E-03

2.02E-03

$2.15 \mathrm{E}-03$

2.37E-03

2.92E-03

3.03

$3.91 \mathrm{E}-03$

$5.74 \mathrm{E}-03$

$5.90 \mathrm{E}-03$

$6.85 \mathrm{E}-03$

$1.01 \mathrm{E}-02$

$1.24 \mathrm{E}-02$

1.24E-02

1.24E-02

1.29E-02

E-02

1.65E-02

1.94E-02

$3.08 \mathrm{E}-02$

$3.08 \mathrm{E}-02$

19E-02

3.47E-02

3.51E-02

$3.51 \mathrm{E}-02$

4.03E-02

7E-02

4.37E-02

4.37E-02

1.02

1.86

4.11

2.05

1.71

2.44

\begin{tabular}{l}
$1.02 \quad 1.49$ \\
\hline
\end{tabular}

0.14

$-1.90$

$-0.57$

0.34

$-0.31$

$-0.13$

0.21

0.79

$-0.40$

0.28

$-0.11$

$-1.14$

$-0.33$

$-1.70$

0.46

$-0.41$

0.54

$-0.11$

$-0.26$

0.40

$-0.09$

$-2.59$

1.77

3.57

2.07

1.88

$-1.95$

-1.95
1.63

$-1.92$

1.58

1.58
-2.54
1.83

1.83

1.95

1.81
-0.091
-0.56

0.56

$-0.07$

0.01

$-0.36$

0.21

$-0.36$

0.16

$-1.90$

$-2.25$

$-2.17$

$-2.13$

1.51

$-2.14$

$-2.15$

$-1.61$

1.48

-1.61
-3.80

$-1.72$

1.61

1.61
1.90

$-1.64$

1.57

$-0.13 \quad-2.24$
-0.56

$-0.56 \quad-1.64$

1.66

$\begin{array}{ll}0.14 & -2.29 \\ 3.40 & 1.25\end{array}$

\begin{tabular}{l|l}
3.40 & 1.25 \\
\hline
\end{tabular}

$0.06-2.36$

$\begin{array}{ll}0.06 & -2.36 \\ 0.05 & -2.84\end{array}$

\begin{tabular}{|c|c|}
\hline-0.23 & -2.10 \\
\hline 0.07 & 1.63 \\
\hline 0.41 & 1.60 \\
\hline-1.11 & 1.00 \\
\hline 0.12 & -2.36 \\
\hline
\end{tabular}

- ADAMTS1

CORO2A

* DYSF

- LOX

- MT1X

A SLCO2A1

* THAP1

+ TNS3

$\triangle$ TXNIP

+ WISP2

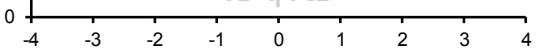

$\log F C$

C

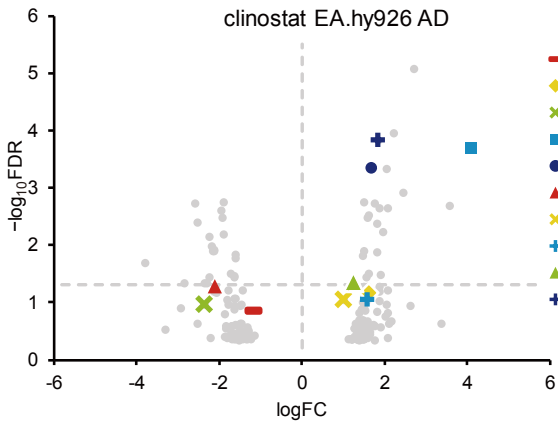

- ADAMTS1

- CORO2A

$\times$ DYSF

- LOX

- MT1X

- SLCO2A1

$\times$ THAP1

+ TNS3

A TXNIP

+ WISP2 
Homologene Symbol P value FDR HUFC EAFC

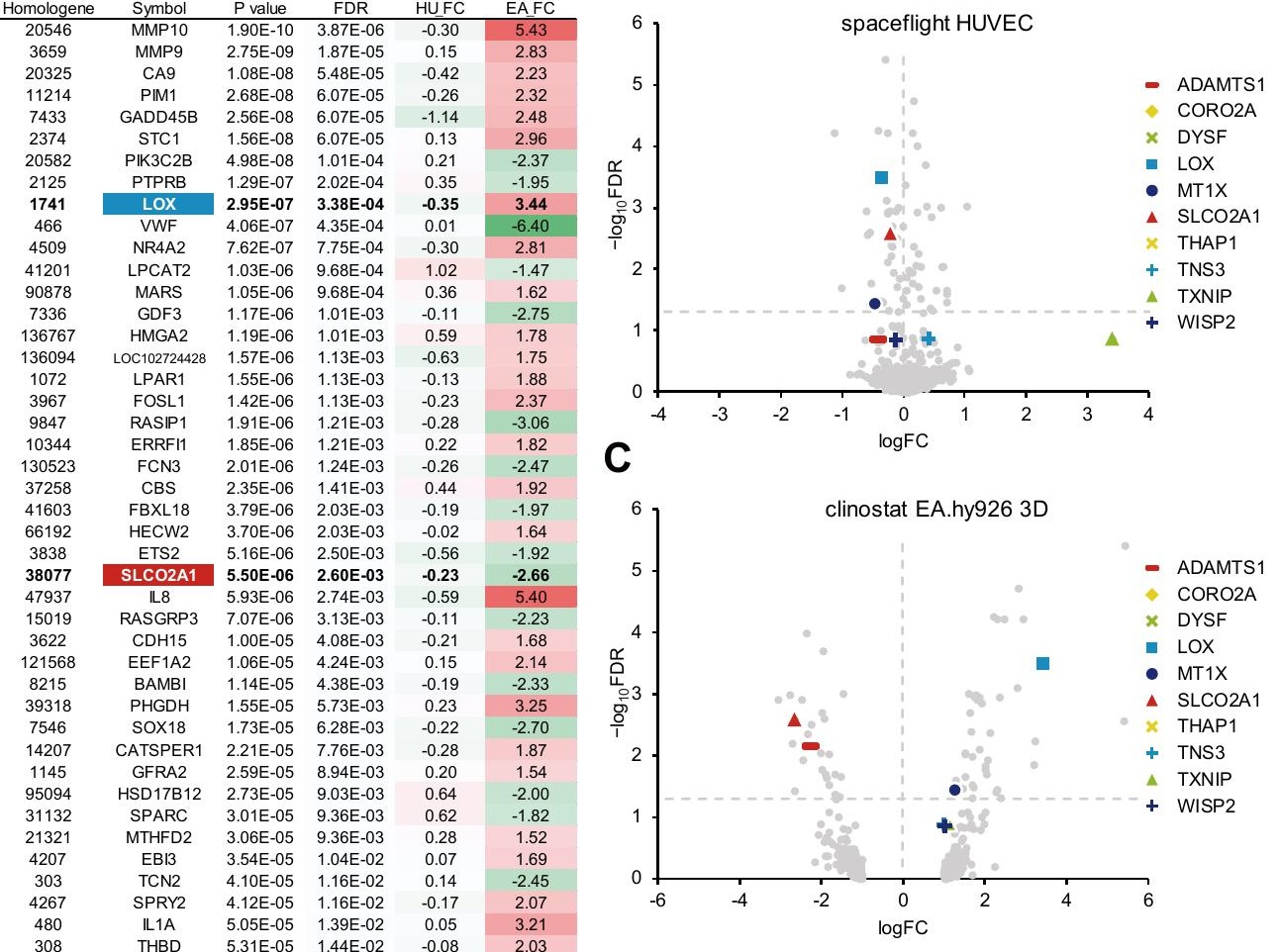

$-0.08$

2.03
31000

9482

55480

8728

8639

68110

49706

20939

1744

121891

1641

7390

2645

502

75163

105490

130542

32049

4742

10764

1424

38186

21381

49713

2882
THBD 5.31E-05 1.44E-02

PTGS2 6.46E-05 1.69E-02

SLCO4A1 6.71E-05 1.71E-02

ALDH2 7.94E-05 1.97E-02

CARD10 8.08E-05 1.98E-02

PPP1R15A 8.27E-05 2.00E-02

RPL37 9.20E-05 2.20E-02

LMBR1 9.41E-05 2.22E-02

EPHB4 9.95E-05 2.25E-02

LRP1 1.15E-04 2.55E-02

ERMP1 1.42E-04 3.01E-02

IGFBP6 1.44E-04 3.02E-02

JUNB $\quad 1.65 \mathrm{E}-04 \quad 3.31 \mathrm{E}-02$

LTBP4 1.76E-04 3.48E-02

IL6 1.82E-04 3.52E-02

SHANK3 1.98E-04 3.80E-02

CXCL2 2.01E-04 3.83E-02

MT1X

2.08E-04

$\mathrm{NOTCH} 1$

2.42E-04

MCAM 2.71E-04

NRIP3

2.79E-04

CRYM

2.95E-04

3.83E-02

$4.32 \mathrm{E}-02$

$4.75 \mathrm{E}-02$

$4.82 \mathrm{E}-02$

4.97E-02

$-0.55$

0.14

0.23

$-0.12$

$-1.03$

0.05

0.69

0.52

0.69

0.27

$-0.02$

$-0.38$

0.70

$-0.36$

0.05

$-0.57$

$-0.45$

0.43

0.46

$-0.25$

$-0.09$

2.03

$-1.97$

$-1.88$

$-1.66$

2.05

1.54

$-1.53$

$-1.85$

1.30

$-1.81$

1.69

1.72

1.48

2.32

$-2.66$

2.27

1.32

$-1.67$

$-1.54$

2.39

1.71

\begin{tabular}{|c|c|c|}
\hline TXNIP & $3.07 \mathrm{E}-04$ & $5.07 \mathrm{E}-02$ \\
\hline ADAMTS1 & $6.43 \mathrm{E}-02$ & $5.40 \mathrm{E}-01$ \\
\hline TNS3 & $1.49 \mathrm{E}-01$ & $6.28 \mathrm{E}-01$ \\
\hline WISP2 & $5.66 \mathrm{E}-01$ & $8.53 \mathrm{E}-01$
\end{tabular}

\begin{tabular}{|c|c|}
\hline 3.40 & 1.15 \\
\hline-0.41 & -1.16 \\
\hline 0.41 & 1.00 \\
-0.13 & 1.04 \\
\hline
\end{tabular}

B 
homologene

Symbol

$P$ value FDR

soleus vastus I vastus |

leus vastus / gcnemius soleus EDL gcnemius calf tonue longis

4038

$\begin{array}{lll}\text { LRP4 } & 2.92 \mathrm{E}-11 & 3.33 \mathrm{E}-09\end{array}$

$\begin{array}{lll}0.54 & 0.33 & 0.28\end{array}$

$0.44 \quad 1.15$

0.54

0.00

0.05

0.94

0.71

56262

23503

23371

5111

28951

5902

4487

LRRC8A 2.21E-05 3.54E-04

$\begin{array}{lll}0.10 & 0.11 & 0.29\end{array}$

0.03

0.59

0.31

0.31

0.18

0.95

0.83

ZFYVE26 3.85E-06 8.15E-05

$\begin{array}{lll}-0.20 & 0.25 & 0.35\end{array}$

$-0.19$

0.50

0.33

0.23

0.09

0.31

0.47

$\begin{array}{lllllllll}\text { TNS2 } & 1.34 \mathrm{E}-04 & 1.52 \mathrm{E}-03 & 0.21 & 0.40 & 0.07 & 0.24 & 0.26\end{array}$

$-0.06$

0.09

0.35

0.74

0.02

0.30

0.41

$\begin{array}{lllllllll} & \text { 3.33E-01 } & 5.56 \mathrm{E}-01 & -0.16 & -0.14 & 0.02 & -0.55 & -0.37\end{array}$

$-0.14$

$-0.09$

RANBP1 $1.49 \mathrm{E}-03 \quad 1.06 \mathrm{E}-02 \quad-0.40$

$-0.64 \quad 0.19$

$-0.88$

$-0.61$

0.02

$-0.12$

$-0.16$

-0.04
-0.36

$-0.21$

0.31

0.11

\begin{tabular}{l|l|l|l|} 
MSX1 & $1.56 \mathrm{E}-02$ & $6.57 \mathrm{E}-02$ & -0.56
\end{tabular}

$-0.21$

$-0.28$

$-0.37$

$-0.40$

0.03

$-0.65$

$-0.11$

$-0.08$

$-0.33$

$-0.10$

THBS1

$9.34 \mathrm{E}-06 \quad 1.72 \mathrm{E}-04$

$\begin{array}{ll}-0.09 & -0.49\end{array}$

$-0.26$

$-1.15 \quad-0.82$

$-0.72$

$-0.81$

$-0.69$

$-0.47$

$-0.05$

$-0.01$

$-0.40$

$-0.18$

$-0.17$ 


\section{$\mathbf{A}$}

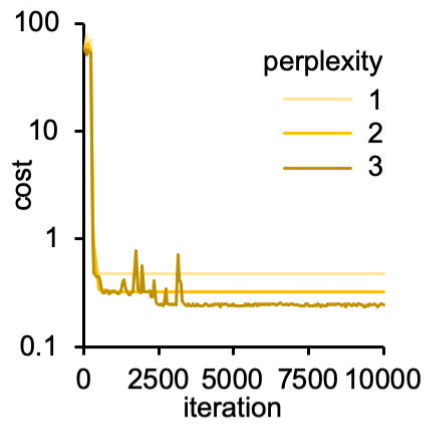

E

$$
\text { E }
$$$$
10
$$

0.1 iteration
B $\quad$ C

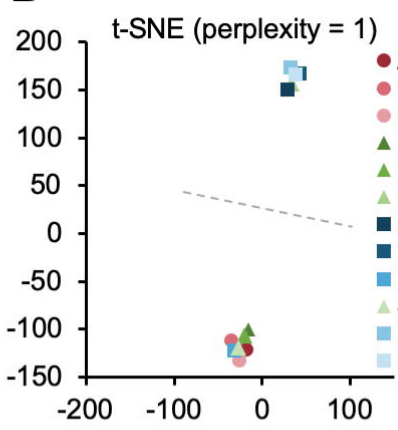

F

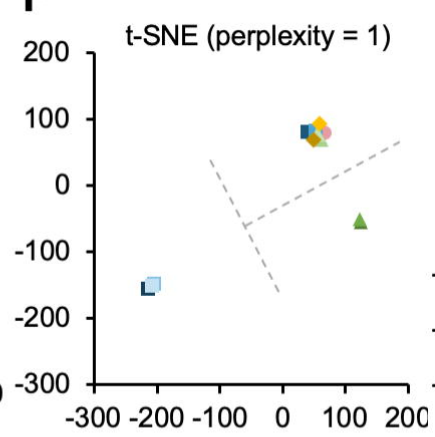

$200{ }^{\mathrm{t}-\mathrm{SNE}}$ (perplexity $\left.=2\right)$

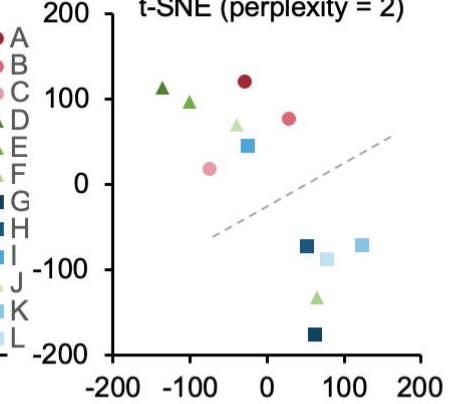

G

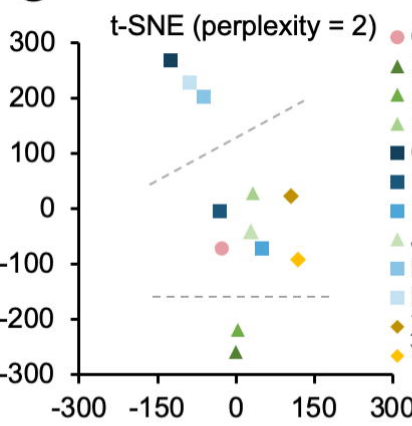

D

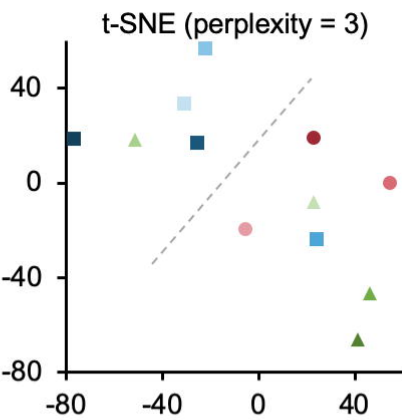

H

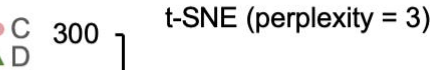

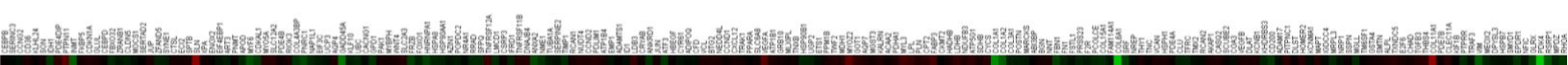

I

-A

- B

$\mathrm{C}$

$\Delta \mathrm{D}$

$\triangle E$

J

-

$\mathrm{H}$

$\triangle F$

$\mathrm{K}$

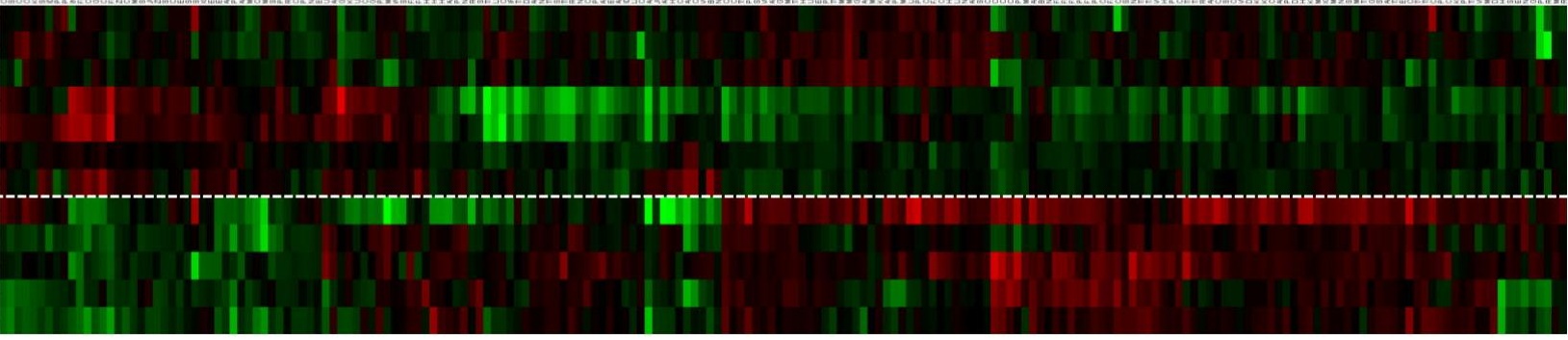




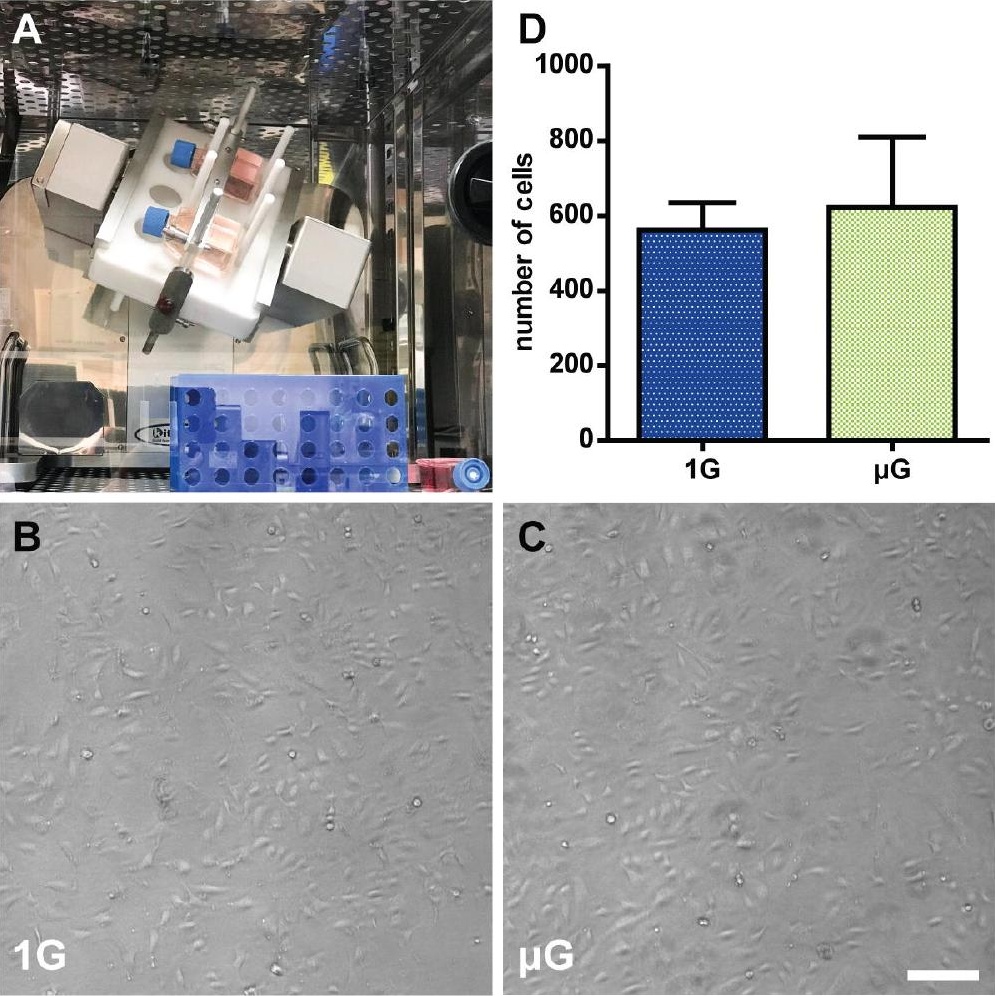


$A_{4}$

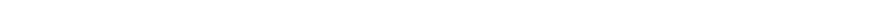

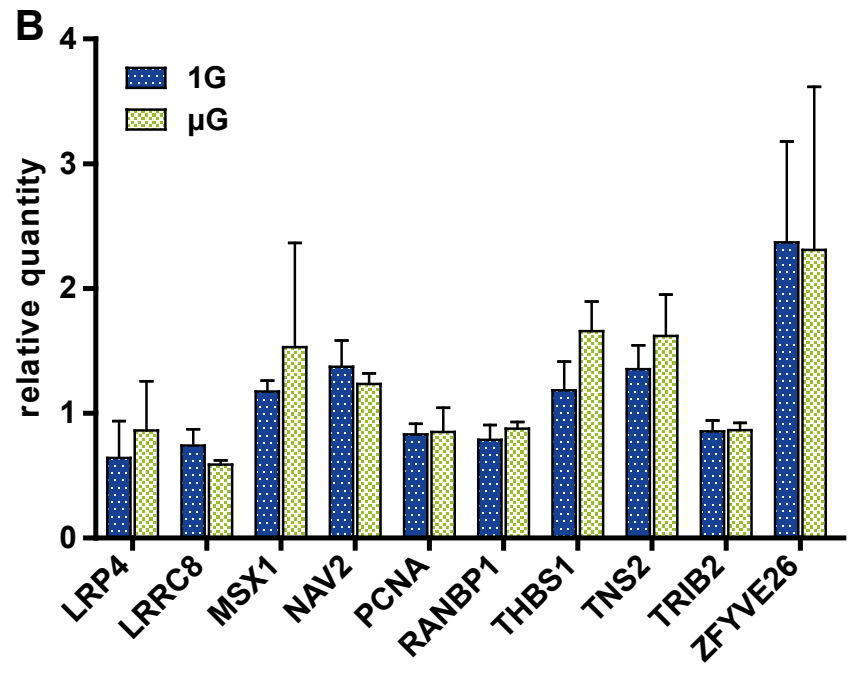




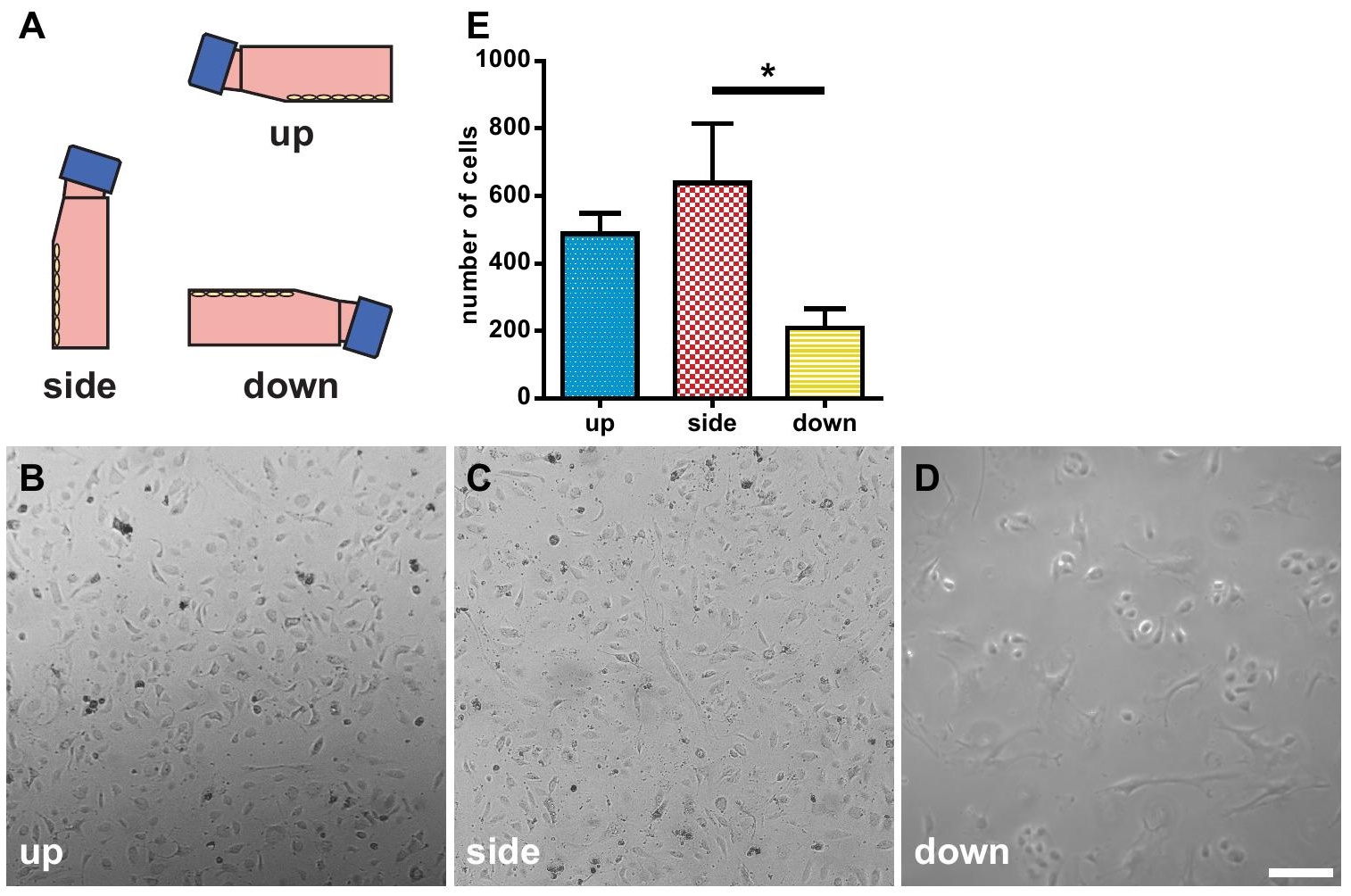




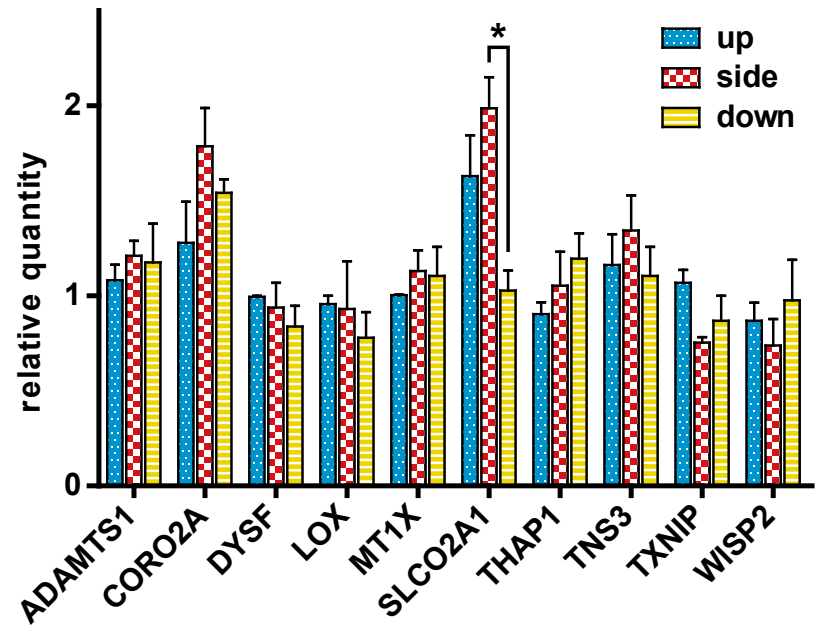

